\title{
Le discours direct entre guillemets sans verbe introducteur dans les textes traduits du japonais
}

\author{
Takagaki Yumi \\ Université préfectorale d'Osaka \\ takagaki@lc.osakafu-u.ac.jp
}

\section{Préliminaires}

Cet article traite du discours direct entre guillemets sans verbe introducteur. On partira d'un exemple emprunté à un conte de Natsume Sôseki ${ }^{1}$. (Dans cet article, les caractères en gras sont de notre fait.)

\footnotetext{
«C'est là, c'est là ! juste au pied de ce cryptomère!" dit la voix qui résonna clairement à travers le rideau de la pluie. Je m'arrêtai malgré moi, et me retrouvai dans les profondeurs de la forêt. À quelques pas, je distinguai une forme noire : c'était sûrement l'arbre indiqué par le gamin.

«Père, c'était bien au pied de ce cryptomère, n'est-ce pas ? "

Je ne pus m'empêcher d'acquiescer.

«C'était la cinquième année de l'ère de Bunka, l'année du Dragon, n'est-ce pas? » Cela se passait effectivement en ce temps-là.

« Eh bien ! voilà exactement cent ans que tu m'as assassiné !»
}

Les premières paroles entre guillemets ( $C$ 'est là, $[\ldots] »)$ sont accompagnées d'un verbe dit, alors que les trois suivantes, transcrites en gras ( Père, $[. .]$.$» ; " C'était [. .]$.$» ; " Eh bien! [...]»), sont introduites$ sans verbe d'énonciation. Ce sont ces formes qui nous intéresseront, à partir de textes littéraires traduits du japonais. Le choix n'est pas arbitraire. Notre réflexion prend son départ dans les écarts observés en matière de discours rapporté entre certaines traductions françaises et les textes originaux. On pourrait attribuer ces différences à des variantes purement stylistiques, à un choix opéré par les traducteurs si nous n'avions rencontré une pluralité d'exemples dans des œuvres écrites par des auteurs traduits par différents traducteurs de sorte que la réécriture paraît plus ou moins systématique. Elle pourrait expliciter les possibilités de textualisation en français.

Dans ce qui suit, nous verrons d'abord dans la section 2 quelques travaux parmi les plus récents et nous présenterons une conception continuiste du discours rapporté. Dans la section 3, nous expliquerons les caractéristiques des textes français traduits du japonais. Dans la section 4, nous observerons d'abord dans quatre textes traduits du japonais des caractéristiques du discours rapporté du français avant de mettre en lumière les conditions qui favorisent, en français, l'apparition d'une forme de discours direct entre guillemets sans verbe introducteur. Ce travail s'inscrit dans la perspective ouverte par Takagaki (2011) en établissant les liens des formes de représentation textuelles et de l'instance d'énonciation conçue dans sa définition anthropologique, culturelle.

\section{Le continuum dans le discours rapporté}

Les études sur le discours rapporté en distinguent traditionnellement trois formes : le discours direct, le discours indirect et le discours indirect libre. À ces trois formes, qui ont été abondamment analysées par les grammairiens, s'est ajoutée au début du siècle dernier une quatrième forme : le discours direct libre qui n'a pas de marqueurs explicites tels qu'un verbe introducteur et des guillemets. La plupart des études classiques ont divisé le discours rapporté en plusieurs catégories aux propriétés bien distinctes. Or les travaux les plus récents ont rapporté l'existence de plusieurs formes mixtes, telles que le discours indirect mis entre guillemets (Authier 1993) et le discours direct avec que (Bruca Cuevas 1996). Ces formes hybrides ne se laissent pas facilement ramener à la distinction nette entre discours direct et discours indirect (libre). Cette irréductibilité indique l'insuffisance de la démarcation tranchée des phénomènes 
concernés. Pour intégrer cette hétérogénéité, certains linguistes ont proposé une vision graduelle de l'organisation du discours rapporté (Rosier 1999, 2008; Charaudeau et Maingueneau 2002; Maingueneau 2005, 2010). Dans cette interprétation en continuum, Rosier (2008) a proposé d'illustrer onze formes citées ci-dessous. (DIL = discours indirect libre; $\mathrm{DI}=$ discours indirect $; \mathrm{DD}=$ discours direct $; \mathrm{DDL}=$ discours direct libre.)

1. Il n'arrêtait pas de parler de sa maladie, qui le hantait (relative de DIL)

2. $\quad$ Il n'arrêtait pas de parler : sa maladie le hantait (DIL narratif)

3. Il n'arrêtait pas de parler: nom de nom, comme sa maladie le hantait! (DIL mimétique)

4. Il n'arrêtait pas de dire que sa maladie le hantait (DI)

5. Sa maladie le hantait, ne cessait-il de répéter (DI avec incise)

6. Il n'arrêtait pas de dire que « sa maladie le hantait ! (DI mimétique)

7. Il n'arrêtait pas de dire « sa maladie le hantait !» (DI sans que)

8. Il n'arrêtait pas de dire que « ma maladie me hante ! » (DD avec que)

9. Il n'arrêtait pas de parler : « ma maladie me hante !» (DD)

10. Il n'arrêtait pas de parler ma maladie me hante! (DD émancipé typographiquement)

11. Il la regarda. Ma maladie me hante ! (DDL)

D'après Rosier, cette liste n'est pas exhaustive. En effet, la forme qui nous intéresse n'y figure pas même si nos exemples doivent être considérés à l'intérieur de cette interprétation en continuum.

Tout comme Rosier, Maingueneau $(2005,2010)$ a proposé une double échelle comprenant six degrés du discours rapporté, correspondants à autant d'instances narratrices. Les voici :

Discours rapporté

Mimétisme minimal entre discours citant et discours cité

Degré 1 : discours narrativisé

(Il raconta son enfance difficile.)

Degré 2 : discours indirect

(Il lui dit qu'il avait eu une enfance difficile.)

Degré 3 : discours indirect contaminé lexicalement ou avec îlot textuel

(Il lui dit qu'il avait eu une enfance « difficile », que sa vie avait été « un tunnel sombre ».)

Degré 4 : discours indirect libre d'un $\mathrm{MQC}^{2}$

(Ils lui parlaient de leur enfance. Elle avait été si difficile! ils avaient dû travailler très jeunes...)

Degré 5 : discours indirect libre d'un personnage

(Paul songeait à son enfance. Qu'elle avait été difficile! Il ne pouvait y penser sans souffrir. Un jour ou l'autre il faudrait bien qu'il se libère de ce souvenir.)

Degré $6:$ discours direct d'un personnage

(Paul ne cessait de parler: " J'ai eu une enfance difficile, très difficile. Je me demande comment j'ai pu tenir le coup. »)

Mimétisme maximal

Instances narratrices

Distance maximale entre la manière de parler du narrateur et celles des personnages

Degré 1 : narrateur zéro (= narrateur anonyme au langage non-marqué socialement)

Degré 2 : narrateur zéro avec contamination lexicale

Degré 3 : narrateur-témoin indéterminé

Degré 4 : narrateur-témoin qui se pose en je

Degré 5 : narrateur-MQC ${ }^{3}$

Degré 6 : narrateur qui est un personnage individualisé de l'histoire

Distance minimale

Mangueneau synthétise les divers types de discours rapporté et de narration dans cette double échelle, où sont placés aux extrêmes opposés les deux pôles du dispositif narratif: la narration zéro (degré 1), totalement extérieure au monde évoqué, et le récit fait par un personnage identifié de l'histoire (degré 6). Cette classification de Maingueneau nous est utile parce qu'elle intègre les instances narratrices qui seront 
employées dans notre analyse. Nous verrons que les textes français et les textes japonais sont le plus souvent différents en matière d'instances narratrices, en particulier lorsqu'il s'agit des paroles intérieures.

La forme que nous traitons dans cet article n'est pas accompagnée de verbe introducteur. Par le verbe introducteur, nous entendons un verbe d'énonciation tel que (se) dire, parler, répondre, questionner, ajouter, s'adresser, (se) demander, proposer, répéter, penser ${ }^{4}$. Cette énumération n'est pas exhaustive. Lors d'une citation, le verbe introducteur apparaît typiquement sous forme de «X DIT » où $X$ indique le locuteur et DIT un verbe d'énonciation. Les exemples cités dans la liste de Rosier et celle de Maingueneau sont tous accompagnés de cette forme. Or, ce qui nous nous intéresse est le discours direct sans «X DIT ».

Nous allons essayer de préciser les conditions d'apparition du discours direct entre guillemets sans verbe introducteur, forme peu étudiée et qui ne figure pas dans les énumérations citées. Or, «X DIT » permet d'identifier l'instance narratrice. Son absence avant ou après un discours rapporté n'est possible que lorsqu'il n'y a pas de confusion en matière d'émetteur des paroles citées. Pour éviter toute interprétation erronée, il doit y avoir une stratégie textuelle pour guider le lecteur. Cette stratégie peut varier d'une langue à une autre. La caractéristique de la stratégie du français sera mise en lumière au moyen de notre étude basée sur les textes traduits du japonais.

\section{Les textes traduits du japonais}

Beaucoup d'études sur le discours rapporté ont choisi leurs exemples dans les productions littéraires. Si ces textes illustrent clairement diverses alternatives d'expression en langue française, et leur relative complexité, une étude de cas portant sur des œuvres risquerait de nous éloigner de l'analyse proprement linguistique, en affectant l'étude du style d'un écrivain en particulier. Par ailleurs, la problématique retenue n'entend pas se cantonner à une application stylistique au sens étroit du terme. En prenant comme objet d'observation des traductions françaises d'œuvres littéraires japonaises, notre objectif n'est pas l'étude contrastive de deux langues, mais la mise en évidence de cas périphériques actualisés par le français. En effet, le japonais dispose de formes de discours rapporté peu exploitées en français ${ }^{5}$. Dans la traduction, la volonté de se montrer fidèle à l'original aboutit parfois à un français moins « naturel », qui permet pourtant de mettre en évidence des usages peu courants dans des exemples attestés.

Le français et le japonais connaissent deux modes fondamentaux de discours rapporté : le mode direct et le mode indirect. Pourtant, les procédés syntaxiques employés dans chacune de ces deux langues pour distinguer ces deux modes sont très différents. De plus, la traduction de diverses formes de discours rapporté est encore complexe parce qu'elles ne revêtent pas la même signification d'une langue à l'autre : ainsi, en japonais, il est tout à fait courant que les éléments déictiques soient présents dans le discours indirect et que la traduction française les actualise dans le discours citant. En japonais, il n'y a pas de concordance des temps entre le temps du verbe de la proposition principale et celui de la proposition subordonnée. De plus, le sujet de la phrase n'est pas obligatoirement exprimé ; le plus souvent, il demeure implicite et une règle par défaut s'applique qui comble cette lacune par un thème textuel. Celui-ci est le plus souvent un élément saillant dans le cotexte amont ou bien le locuteur je, qui fonctionne comme un thème par défaut. Comme le disent Li et Thompson (1976), le japonais est une langue où la relation grammaticale thème-rhème (topic-comment) joue un rôle aussi important que la relation sujet-prédicat, qui est primordiale pour les langues comme le français. Tout se passe comme si les inférences sémantiques et pragmatiques suppléaient en japonais ce que la connexion syntaxique décide en français. Il y a à cela une raison structurale, inscrite dans la formulation requise par l'interaction.

En outre, la distance entre l'oral et l'écrit est variable d'une langue à l'autre, ce qui peut expliquer certaines variantes de traduction et la nécessité de prendre en compte le contexte avant d'effectuer le choix de la traduction d'une forme de discours rapporté, une conversion très fréquente lors de la traduction française des œuvres littéraires en japonais.

Ces différences entre les deux langues donnent une influence sur les conditions de l'apparition du discours direct entre guillemets sans verbe introducteur. Par exemple, le français a souvent recours à la 
typographie dans l'identification des instances narratrices alors que le japonais emploie une stratégie pragmatique explicitée lexicalement. D'après la convention typographique française, le tiret $(-)$ marque le changement d'interlocuteur dans les dialogues, avec l'alinéa et le plus souvent en combinaison avec les guillemets. Dans les dialogues, on peut, soit placer les guillemets ouvrants au début de la première réplique et les guillemets fermants à la fin de la dernière réplique, soit se passer de guillemets et n'utiliser que des tirets. Bref, en français, le tiret joue un rôle similaire aux guillemets dans le phénomène du discours rapporté. Or, le japonais ne dispose pas d'une telle marque signalant une alternance du locuteur : si deux segments entre guillemets japonais $([\quad \mid$ ) sont juxtaposés (éventuellement avec une brève incise qui ne contient pas de verbe introducteur), cette séquence peut être interprétée soit comme suite des paroles émises par un seul locuteur, soit comme échange entre deux personnes $(c f$. $(3 \mathrm{~b}))$. La convention de la typographie japonaise ne permet pas de distinguer ces deux cas.

Par ailleurs, en japonais, des formes d'expressions linguistiquement codées spécifient l'âge, le sexe et le statut social du locuteur. L'emploi ou le non-emploi de ces expressions indiquent quelle relation interpersonnelle entretiennent les interlocuteurs. Ces indices facilitent l'assignation de celui qui prend la parole dans l'échange.

Prenons un exemple pour illustrer cette différence entre les deux langues. Le texte suivant emprunté à un conte de Tsushima Yûko ${ }^{6}$ présente un dialogue entre un mari et sa femme, laquelle est aussi la narratricetémoin qui se pose en je (degré 4 selon l'échelle des instances narratrices de Maingueneau, citée dans la section 2).

(2) Il y avait une maison avec un petit cyprès planté à côté de l'entrée. Cinq marches de pierre menaient à une porte peinte en bleu pâle. Le cyprès poussait dans un interstice de moins d'un mètre entre les marches et la porte. Ses branches cachaient une fenêtre en saillie dont le cadre était peint de la même couleur que la porte.

«C'est pas mal! dit joyeusement mon mari. cerisier...

- Oui, mais je n'aime pas cet arbre. Si ç'avait été un magnolia ou un

— Mais c'est bien plus distingué, un cyprès. »

C'était une maison à un étage.

En ce qui concerne la présence et l'absence de la forme «X DIT », la traduction française est fidèle à l'original japonais : les premières paroles entre guillemets («C'est pas mal!) sont accompagnées de cette forme (dit joyeusement mon mari), alors que celle-ci manque dans les suivantes, transcrites en gras (Oui, mais [...]; - Mais c'est bien plus distingué, un cyprès.). Malgré l'absence de cette forme, dans ce passage, il n'y a pas de confusion sur l'identification des émetteurs des paroles. La première phrase entre guillemets est explicitement attribuée au mari. Le premier tiret indique que les paroles qui le suivent (Oui, mais [...]) doivent être attribuées à sa femme. Comme il n'y a que deux personnages qui se parlent, le second tiret indique que les paroles qui le suivent (Mais c'est bien plus distingué, un cyprès.) ne peuvent êtres émises que par le mari.

Or, comme le japonais ne dispose pas de marque équivalente au tiret signalant une alternance du locuteur, ce sont les expressions spécifiant des traits du locuteur qui constituent les indices dans l'assignation de celui qui prend la parole dans l'échange. En effet, dans l'original japonais du texte en (2), la phrase correspondante à la dernière phrase émise par le mari (Mais c'est bien plus distingué, un cyprès.) se termine par les particules d'affirmation da-zo, expression typiquement employée par les hommes ${ }^{7}$. L'emploi de cette terminaison attribue pragmatiquement cette phrase au mari. C'est ainsi que le texte japonais évite une interprétation fausse.

Cette différence de stratégie textuelle entre les deux langues peut expliquer des cas d'insertion d'un verbe introducteur qui n'existe pas dans le texte original. Dans l'exemple suivant, extrait d'un conte de Yoshimoto Banana $^{8}$, la traduction française a un verbe d'énonciation dont l'équivalent n'existe pas dans l'original japonais. Il s'agit d'un dialogue entre un homme et une femme, laquelle est aussi la narratricetémoin qui se pose en je (degré 4 selon l'échelle de Maingueneau). La première phrase entre guillemets 
("C'est là que j'habite,) est accompagnée du verbe dire, alors que le reste des paroles après le tiret (Quelle chance! [...]) est introduit sans verbe d'énonciation.

(3a) Au retour, nous sommes passés par le parc. Entre les arbres, on voyait l'immeuble des Tanabe.

« C'est là que j'habite, ai-je dit avec un geste de la main.

— Quelle chance! C'est juste à côté du parc. À ta place, je me lèverais à

cinq heures du matin pour me promener. »

Il a ri.

Or, le texte original japonais n'a pas de verbe introducteur correspondant à dit. Une traduction plus fidèle à l'original serait comme suit :

(3b) $\mathrm{Au}$ retour, nous sommes passés par le parc. Entre les arbres, on voyait l'immeuble des Tanabe.

"C'est là que j'habite. »

J'ai fait un geste de la main.

«Quelle chance ! C'est juste à côté du parc. À ta place, je me lèverais à cinq heures du matin pour me promener. »

Il a ri.

En tant que texte français, s'il n'y a pas d'expression ai-je dit, l'enchaînement textuel est beaucoup moins naturel : en (3b) il est plus difficile de déterminer qui a dit quoi qu'en (3a). Or, dans le texte original japonais, il n'y a aucune ambiguïté grâce à certains indices lexicaux. Les premières paroles citées se terminent par des particules no-yo, terminaison typiquement employée par les femmes. Les secondes paroles citées contiennent ore, un pronom de la première personne qui n'est employé que par les hommes. Ces marques excluent pragmatiquement une fausse attribution des paroles aux instances narratrices.

Les exemples (2) et (3) illustrent la dissymétrie de ce que représentent des guillemets et un tiret qui n'est jamais qu'une convention orthographique, par rapport à l'absence d'un verbe introducteur qui se situe sur le plan syntaxique. Dans la section suivante, nous examinerons des cas de textes français qui ne s'expliqueraient pas de la même manière.

\section{Le discours direct entre guillemets sans « X DIT » en français}

\subsection{Des paroles d'un personnage au lieu d'une description}

L'exemple suivant extrait d'un roman de Yasunari Kawabata traduit une forme de discours rapporté par une autre forme en français. La traduction française citée en $(4 a)^{9}$ emploie une expression condensée, intrigué, pour décrire la réaction mentale du personnage, tandis que dans le texte original japonais, traduit plus littéralement en (4b), la pensée est présentée directement par l'expression "Qu'est-ce que c'est? ", mise entre guillemets sans verbe introducteur.

(4a) Un jour qu'il était venu voir Eguchi chez lui, il avait aperçu une chose rouge tombée sur la mousse du jardin que flétrissait l'automne et, intrigué, il était allé aussitôt la ramasser. C'était la baie rouge d'un aucuba. Il y en avait un certain nombre, dispersées un peu partout.

(4b) Un jour qu'il était venu voir Eguchi chez lui, il avait aperçu une chose rouge tombée sur la mousse du jardin que flétrissait l'automne et, "Qu'est-ce que c'est ? », il était allé aussitôt la ramasser. C'était la baie rouge d'un aucuba. Il y en avait un certain nombre, dispersées un peu partout.

La rupture introduite par la traduction (4b), si étrange qu'elle paraisse, demeure compréhensible car il y a un seul personnage saillant, désigné par $i l$, et le lecteur lui attribue automatiquement les paroles rapportées. Il n'y a pas d'ambiguïté. Mais la logique de la textualisation en français exigerait, par exemple, l'insertion d'une incise comme dit-il ou pensa-t-il après "Qu'est-ce que c'est? ". L'étrangeté textuelle de (4b) est étroitement liée aux conventions littéraires de la langue française. 
On notera l'emploi d'aperçu juste avant "Qu'est-ce que c'est? ». Ce verbe de perception justifie dans le texte japonais la présence du discours direct sans verbe introducteur du type dire ou penser ${ }^{10}$. La mention d'un verbe de perception induit le lecteur à conjecturer qu'il y a quelqu'un qui perçoit, même si le sujet de cette sensation reste implicite. (Le japonais est une langue pro-drop, la présence du sujet grammatical est facultative.) En français, l'actualisation d'un verbe de perception peut n'être pas suffisante pour introduire un discours direct entre guillemets.

\subsection{Les paroles intérieures 1}

L'exemple suivant sans guillemets indique une piste concernant les conditions sur l'apparition des guillemets avec le discours rapporté. Si le cotexte amont décrit à suffisance la psychologie d'un personnage du récit, le discours direct sans verbe introducteur est licite. À la différence de (4a), en ce qui concerne la restitution du discours rapporté, la traduction de Nagai Kafû citée en $(5)^{11}$ se révèle en tous points conforme à l'original. Dans ce texte, le personnage principal, Ragetsu, désigné dans la première phrase du texte par $i l$, pense à son neveu Chôkichi.

(5) Et en particulier, s'il se remémorait l'histoire de son passé, il n'avait plus besoin d'interroger Chôkichi pour lire clairement jusqu'au tréfonds de son cœur. Le temps de sa jeunesse, quand il travaillait accroupi derrière le comptoir de l'obscur magasin de prêts sur gages légué par les ancêtres, sans pouvoir profiter des radieuses journées de printemps, quelle peine, quelle pitié ç'avait été ! Et plutôt qu'à inscrire, sous la morne flamme de la lampe, les entrées et les sorties d'argent sur le grand livre de comptes, quel plaisir avait-il goûté à lire quelque « livre d'esprit » à l'étage lumineux d'une petite maison au bord de la rivière ! Chôkichi déclare que, plutôt que de devenir une espèce d'austère employé à moustaches, il préfère traverser l'existence en pratiquant les arts qu'il aime ? Eh bien, il y a là une vie et ici une autre ! Ragetsu cependant, dans la mesure où il avait été promu, sans qu'il y eût moyen de s'y soustraire, au rang de conseilleur, ne pouvait se permettre de dévoiler jusqu'à ce point ses sentiments personnels; c'est pourquoi il avait dû se contenter de gratifier son neveu de bonnes paroles de circonstance, comme il l'avait fait pour la mère.

Les deux phrases en gras ne sont ni mises entre guillemets, ni précédées par une locution introductive. Néanmoins, le lecteur est induit à les attribuer à la pensée de Ragetsu, une interprétation imposée grammaticalement. Les verbes conjugués sont au présent et nos connaissances extralinguistiques nous indiquent qu'il ne s'agit pas d'un présent de vérité générale. Cette rupture temporelle introduit une rupture avec les deux phrases au passé qui l'encadrent. (Dans l'original japonais, les deux phrases sont au présent.) Ensuite, la ponctuation signale également qu'il s'agit du discours direct libre. La première phrase se termine par un point d'interrogation, ce qui serait difficile s'il ne s'agissait pas d'une pensée de Ragetsu. De plus, la possibilité d'insertion de certaines expressions confirmerait notre observation. Le discours direct libre peut présenter des personnes de l'interlocution: je, tu, nous, vous. Or dans cette séquence, l'intrusion d'une première personne dans la première phrase est possible : Chôkichi me déclare que, [...]; Chôkichi nous déclare que, [...]. Enfin, on pourrait insérer des éléments « oralisants » tels que Oh!, Ah! au début de ces phrases : Oh ! Chôkichi déclare que, [...]; Ah! Chôkichi déclare que, [...].

Nakayama (1990) affirme que c'est le texte original qui a décidé de la traduction de ces deux phrases en (5). Dans le texte français, l'enchaînement en (5) n'est pas dissonant en ce sens que, quelles que soient les marques de l'énonciation, suivre le discours rapporté ne présente aucune difficulté. Par ailleurs, si l'on insérait entre guillemets les deux phrases en gras en (5), l'enchaînement textuel paraîtrait moins naturel, ou bien il faudrait compenser l'artifice typographique en insérant un verbe du type se dire ou penser. Sans cela, le discours direct entre guillemets pourrait sembler impossible. Cependant l'exemple suivant montre que ce n'est pas toujours le cas. 


\subsection{Les paroles intérieures 2}

À la différence de l'exemple (5), qui décalquait l'original dans le recours au mode de discours, la traduction de La Femme des sables d'Abe Kôbô ${ }^{12}$ a adopté une présentation très différente (c'est nous qui numérotons les phrases).

(6a) [1] Le corps raidi, la femme s'enferma dans un long silence, maintenant seulement le parapluie sous lequel l'homme mangeait. [2] Lui, sous le sentiment qu'elle le pressait, finit son repas sans dire un mot. [3] Sur le dessus du parapluie, on eût aisément, du doigt tracé des caractères, telle était la couche de sable qui, déjà, le recouvrait.

[4] Et puis, cette insupportable humidité !...

[5] « Mais non, enfin, [6] raisonnait l'homme à part lui. [7] Mais non, ce n'est pas dans le sable qu'est l'humidité : [8] c'est mon corps qui est humide, mon seul corps à moi ! »

[9] Par-dessus le toit, le vent gémissait. [10] Il voulut retirer de sa poche ses cigarettes: [11] il trouva sa poche pleine de sable; [12] et avant même d'allumer sa première cigarette, il lui parut certain que le goût en serait âcre.

[13] De son flacon à tuer ses prises, il sortit un insecte, [14] et, avant que la bête ne se fît rigide, la fixa avec des épingles :

[15] « Essayons de préserver au moins la forme des pattes : je vais les disposer par paires...»

[16] Du dehors, de là où se trouvait l'évier, le bruit lui parvenait de la vaisselle que la femme lavait... [17] Cette maison, au fait, n'y avait-il personne d'autre à l'habiter ? [18] Il se posait la question.

[19] La femme revint, et, en silence, dans un coin de la pièce, se mit à étendre les couvertures.

[20] « Tiens, [21] se dit-il : [22] c'est ici qu'elle fait mon lit! [23] Mais elle, alors ? [24] Où diable a-t-elle l'intention de coucher ? [25] Là-bas, bien sûr, dans la pièce du fond, de l'autre côté de cette natte qui sert de cloison. [26] Ça ne peut être que ça, car, d'apparence d'une troisième pièce, je n'en vois nulle part...[27] Pourtant, vrai, recevoir un hôte, se garder à soi-même la pièce $\mathrm{du}$ fond et cantonner le visiteur dans la pièce près de l'entrée... vrai, c'est une drôle de manière de faire, et tellement contraire à l'usage ! [28] À moins que... à moins que, dans cette pièce du fond, ne soit justement couché quelqu'un de très malade, incapable de bouger: ce serait l'explication la plus naturelle...[29] Bah, le plus clair n'est-il pas que, pour une femme qui vit seule, ça ne doit guère être de règle de se mettre en trop grands frais pour de simples voyageurs de passage !...»

Avant d'aborder le problème du discours direct, nous récapitulons l'ensemble du texte (6a). Il commence par un récit du narrateur zéro ([1]-[3]) ; la phrase [4] est interprétée comme les paroles intérieures de l'homme. Trois segments cités sont mis entre guillemets ([5]-[8], [15] et [20]-[29]). Parmi ces trois citations, qui alternent avec le récit à la troisième personne, la phrase [15] est en discours direct sans verbe introducteur. La phrase [17] est un monologue intérieur de l'homme, en discours indirect libre.

Or la présentation du texte original est très différente. Tout le texte correspondant à (6a) est une suite de phrases sans aucuns guillemets. Si la traduction est constituée de neuf paragraphes, l'original n'en a que quatre : c'est seulement après les phrases correspondantes à [3], [12] et [17] que le texte va à la ligne. Bref, le texte japonais se présente comme une masse peu structurée. De plus, en japonais, il n'existe pas de phrases correspondantes à [5], [6], [18], [20] et [21]. Autrement dit, il n'y a pas d'incises ([6] raisonnait l'homme à part lui; [21] se dit-il), ni interjections ([5] Mais non, enfin; [20] Tiens), ni discours narrativisé ([18] Il se posait la question.).

Voici un texte plus fidèle à l'original. ([ø] signifie un élément implicite.)

(6b) [1'] Le corps raidi, la femme s'enferma dans un long silence, maintenant seulement le parapluie sous lequel l'homme mangeait. [2'] Lui, sous le sentiment qu'elle le pressait, finit son repas sans dire un mot. [3'] Sur le dessus 
du parapluie, on eût aisément, du doigt tracé des caractères, telle était la couche de sable qui, déjà, le recouvrait.

[4'] Et puis, cette insupportable humidité !... [7'] Mais non, ce n'est pas dans le sable qu'est l'humidité : [8'] c'est mon corps qui est humide, mon seul corps à moi ! [9'] Par-dessus le toit, le vent gémissait. [10'] [ø] voulut retirer de la poche les cigarettes : [11'] [ø] trouva la poche pleine de sable ; [12'] et avant même d'allumer la première cigarette, il parut certain que le goût en serait âcre.

[13'] Du flacon à tuer les prises, [ø] sortit un insecte. [14'] Avant que la bête ne se fasse rigide, [15'][ø] vais la fixer avec des épingles pour préserver au moins la forme des pattes. [16'] Du dehors, de là où se trouvait l'évier, la femme faisait du bruit en lavant de la vaisselle. [17'] Cette maison, au fait, n'y avait-il personne d'autre à l'habiter?

[19'] La femme revint, et, en silence, dans un coin de la pièce, se mit à étendre les couvertures. [22'] C'est ici qu'elle fait mon lit! [23'] Mais elle, alors ? [24'] Où a-t-elle l'intention de coucher? [25'] Là-bas, bien sûr, dans la pièce du fond, de l'autre côté de cette natte qui sert de cloison. [26'] Ça ne peut être que ça, car, d'apparence d'une troisième pièce, je n'en vois nulle part...[27'] Pourtant, vrai, recevoir un hôte, se garder à soi-même la pièce du fond et cantonner le visiteur dans la pièce près de l'entrée... vrai, c'est une drôle de manière de faire, et tellement contraire à l'usage ! [28'] À moins que... à moins que, dans cette pièce du fond, ne soit justement couché quelqu'un de très malade, incapable de bouger : ce serait l'explication la plus naturelle...[29'] Le plus clair n'est-il pas que, pour une femme qui vit seule, ça ne doit guère être de règle de se mettre en trop grands frais pour de simples voyageurs de passage !...

En matière d'instances narratrices, l'original diffère assez sensiblement de sa traduction. En japonais, les phrases correspondantes à [1'], [2'], [3'] et [13'] sont attribuées au narrateur zéro. Tout le reste du texte serait interprété comme discours direct libre du personnage désigné par l'homme. Par ailleurs, dans la traduction française en (6a), avec plusieurs modes de discours rapporté et non rapporté, les instances narratrices changent entre le narrateur zéro et la position de narration assumée par un personnage individualisé de l'histoire. L'original présente une simplicité énonciative quand la traduction offre de fréquentes alternances.

La traduction en (6a) a recours à des insertions de mots qui ne figurent pas dans l'original : Tiens en [20], diable en [24] et Bah en [29]. D'après Dubois (1989), l'anglais Hey marque une pseudo-citation. Il est possible que son équivalent français Tiens, employé en (6a), ait une fonction similaire. En effet, dans la traduction française de ce roman ${ }^{13}$ sont souvent interpolées des expressions interjectives (tiens, bah, diable, voilà, voyons, oui) sans équivalent dans l'original. La traduction française y recourt pour indiquer au lecteur que quelqu'un raconte l'histoire. Par le truchement de ces expressions, le texte français semble justifier une alternance d'instances narratrices qui n'existait pas dans l'original.

Le rendu de perception (le bruit lui parvenait de la vaisselle que la femme lavait...) de [16] en (6a) serait plus fidèlement traduit comme la femme faisait du bruit en lavant de la vaisselle. En japonais, cette phrase peut être interprétée comme décrite du point de vue du personnage désigné par l'homme. Il suffit d'une expression de perception en japonais pour que cette interprétation soit conjecturée. Or ce point de vue n'est pas possible dans la traduction française où le pronom lui indique la présence d'un narrateur qui n'est pas un personnage individualisé de l'histoire. Il en va de même pour la phrase [9], où le verbe gémir suggère la présence d'un sujet de conscience qui perçoit les sons, de sorte que le lecteur est incité à interpréter la phrase comme décrite à travers le point de vue du personnage.

En comparaison de l'original, les phrases [13] [14] et [15] constituent la principale difficulté. Si l'on prend pour repère la conformité à leur expression en japonais, elles sont très mal traduites. Dans l'original, ne figurent ni guillemets, ni la deuxième moitié de [15] : je vais les disposer par paires. De plus, la phrase originale correspondante à [13'] en (6b) est interprétable comme une description neutre alors que celles correspondantes à [14'] et [15'] en (6b) doivent être comprises comme étant des paroles intérieures du héros. Par ailleurs, en juxtaposant [13] et [14], la traduction française ne respecte pas du tout cette distinction et décrit à tort l'action de « fixer (la bête) avec des épingles » comme un événement 
du passé. Cette erreur de traduction met en relief une caractéristique de la textualisation en français. Le texte français en (6a) distingue clairement le récit à la troisième personne et la pensée du personnage alors que dans le texte japonais, la démarcation n'est pas toujours tranchée entre la partie attribuable à l'hétérodiégétique et celle qui reviendrait au narrateur intradiégétique. En effet, en (6b), en dehors du fait qu'un élément demeure implicite [ø], le texte tel qu'il est réécrit paraît peu naturel du point de vue des instances narratrices.

Si l'on en revient au problème de discours direct du texte (6a), d'après l'observation émise à propos de (4) et (5), le discours direct entre guillemets semblerait exiger un verbe du type dire ou penser. Or, en (6a), la phrase [15] est un discours direct entre guillemets sans verbe introducteur. Pourquoi cette forme, possible en (6a), s'avère impossible en (4b) et en (5) ? Les différences relevées entre ces trois exemples révéleront les conditions qui favorisent son apparition.

Les phrases en question en (4) et (5) introduisent une sorte de rupture. En (4a), avec l'expression intrigué, le héros effectue un déplacement de l'attention, homologue chez le narrateur intradiégétique et le lecteur. En français, le déclenchement ne peut en être des paroles entre guillemets comme "Qu'est-ce que c'est? 》 en (4b). En (5), le texte reproduit d'abord une suite de réflexions sur la vie de Ragetsu. Les phrases en gras (Chôkichi déclare que, [...]) concernent la vie de Chôkichi. On est confronté à l'introduction d'un nouvel élément dans le texte sans qu'on puisse insérer ces deux phrases entre des guillemets. Dans ces deux exemples, les phrases analysées introduisent un changement de perspective ou de thème. Par ailleurs, en (6a), entre la phrase [15] et son cotexte amont, il y a une continuité thématique. Les phrases [13] et [14] décrivent une série d'actions du héros et la phrase [15] explique dans quel but ces actions sont accomplies. Il n'y a aucun élément qui puisse surprendre le lecteur. C'est cette continuité qui semble déclencher l'apparition du discours direct avec guillemets sans verbe introducteur.

Pour tester cette hypothèse, on comparera ces trois énoncés :

(6c) De son flacon à tuer ses prises, il sortit un insecte, et, avant que la bête ne fût rigide, la fixa avec des épingles :

«Essayons de préserver au moins la forme des pattes : je vais les disposer par paires...»

(6d) De son flacon à tuer ses prises, il sortit un insecte, et, avant que la bête ne fût rigide, la fixa avec des épingles :

"Tiens, c'est ici qu'elle fait mon lit!»

(6e) De son flacon à tuer ses prises, il sortit un insecte, et, avant que la bête ne fût rigide, la fixa avec des épingles :

«Tiens, se dit-il, c'est ici qu'elle fait mon lit!»

Le texte $(6 \mathrm{c})$ reproduit partiellement $(6 \mathrm{a})$. Le texte $(6 \mathrm{~d})$ est identique à $(6 \mathrm{c})$, à l'exception de la phrase entre guillemets. Comparé à (6c), les paroles citées sous forme de discours direct en (6d) ne sont pas liées au cotexte amont et leur irruption est brutale. Or, comme le montre l'exemple (6e), cet enchaînement qui pourrait paraître maladroit est nettement amélioré par l'insertion d'une incise : se dit-il.

Bref, en cas de rupture thématique, l'insertion d'une forme de discours direct entre guillemets suppose la présence d'un verbe introducteur. À la différence du japonais où une expression de perception suffit pour justifier l'introduction du discours direct entre guillemets $(c f$. (4)), le texte français tend à exiger une continuité thématique pour pouvoir supporter ce type de présentation.

\subsection{La continuité thématique}

Avant de finir, revenons à l'exemple présenté au début de cet article et reproduit ici comme (1a). Il contient une succession de discours directs entre guillemets. Dans ce texte, le narrateur-témoin qui s'énonce en je porte son fils, le gamin, sur son dos.

(1a) «C'est là, c'est là ! juste au pied de ce cryptomère ! » dit la voix qui résonna clairement à travers le rideau de la pluie. Je m'arrêtai malgré moi, et me 


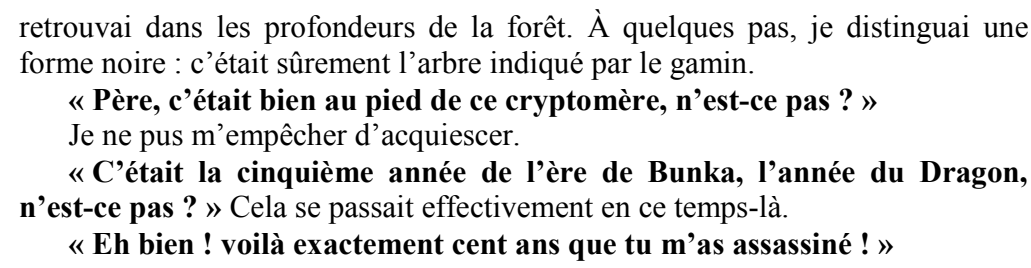

En (1a), il n’y a pas de verbe introducteur pour les trois dernières paroles citées entre guillemets : " Père, $[\ldots] » ;$ " $C$ 'était $[\ldots] »$; «Eh bien ! [...]». Dans la mesure où il s'agit d'un dialogue entre deux personnages et que l'apostrophe Père du deuxième énoncé permet d'identifier le locuteur, il n'y a pas d'ambiguïté concernant le narrateur, même en l'absence de forme «X DIT » qui préciserait le sujet de l'énonciation.

Dans le premier énoncé ( "C'est là, c'est là ! [...]»), le fills parle d'un cryptomère et l'arbre reste un élément important dans la suite du texte, présent dans le deuxième énoncé ( Père, [...]») réalisé comme discours direct sans verbe introducteur. En même temps, dès le début, le fils suggère un événement mystérieux par le pronom cataphorique $c$ '. Si le deuxième énoncé précise le lieu, le troisième (" $C$ 'était [...]») indique l'année de l'événement inconnu du lecteur alors que l'échange entre les deux personnages les présente comme également informés de ce dont il s'agit. Cette continuité justifie l'apparition du troisième énoncé du fils au discours direct entre guillemets sans verbe introducteur. S'il y a un effet de surprise dans son quatrième énoncé, il opère sans la ressource d'une rupture thématique. L'idée d'assassinat, pour terrible qu'elle soit, est le même événement dont il est question depuis le début de l'échange de sorte que les dernières paroles du fils peuvent être présentées sous forme de discours direct avec guillemets sans verbe introducteur.

Nous confirmerons cette observation en modifiant la citation. Au lieu de continuer à parler d'un événement qui a eu lieu au pied d'un cryptomère, en (1b) le fils parle subitement de son poids.

(1b) «C'est là, c'est là ! juste au pied de ce cryptomère!» dit la voix qui résonna clairement à travers le rideau de la pluie. Je m'arrêtai malgré moi, et me retrouvai dans les profondeurs de la forêt. À quelques pas, je distinguai une forme noire : c'était sûrement l'arbre indiqué par le gamin.

«Père, je suis trop lourd?»

Je ne pus m'empêcher d'acquiescer.

« Dans peu de temps, je vais commencer à peser très lourd. »

La pesanteur de l'enfant est un nouveau thème, un élément abrupt qui rompt la continuité thématique du texte. L'enchaînement textuel en (1b) paraît moins naturel que celui en (1a). Avec l'insertion d'une incise : me demanda-t-il, la traduction est plus convenue, plus acceptable.

(1c) «C'est là, c'est là ! juste au pied de ce cryptomère ! " dit la voix qui résonna clairement à travers le rideau de la pluie. Je m'arrêtai malgré moi, et me retrouvai dans les profondeurs de la forêt. À quelques pas, je distinguai une forme noire : c'était sûrement l'arbre indiqué par le gamin.

«Père, me demanda-t-il, je suis trop lourd?»

Je ne pus m'empêcher d'acquiescer.

«Dans peu de temps, je vais commencer à peser très lourd. »

\section{Conclusion}

À partir de textes traduits du japonais, nous nous sommes proposé de déterminer dans quelles conditions pouvait être actualisé un discours direct entre guillemets sans verbe introducteur en français. Cette forme n'est possible que lorsque la continuité thématique est assurée entre les paroles citées et le cotexte amont. Les textes japonais sont plus accessibles à de telles occurrences dans la mesure où la palette des formes d'adresse dans l'interaction et, en deçà, la présence d'une expression de perception constituent des justifications différentes pour son apparition dans un texte. La restriction qui affecte la textualisation 
française, et dont le rapprochement du récit en japonais et de sa traduction en français apporte la démonstration, constitue une caractéristique de la logicisation obligatoire.

Cet article n'est que le point de départ pour une étude générale sur le discours rapporté. Dans notre future recherche, nous pourrions essayer une formalisation des éléments qui licencient ou prohibent tel ou tel usage des autres formes du discours rapporté, tout en avançant une réflexion sur le partage entre ce que produit la syntaxe du français et ce à quoi suppléent sa sémantique et sa pragmatique.

Par ailleurs, le problème de la logique de la textualisation est lié aux conventions textuelles. Si un enchaînement textuel ne semble pas naturel, il s'agit d'une conjecture liée à la nature du pacte de lecture qu'on est conduit à postuler, pacte sous-jacent aux formes de découverte du texte admissibles sans perte de compréhension. En effet, un certain nombre de formes, dont nous avons postulé qu'elles ne seraient pas maladroites en français, seront néanmoins attestées dans des écrits contemporains. Cet aspect conventionnel du texte pourra se raffiner davantage.

\section{Références bibliographiques}

Authier-Revuz, J. (1992). Repères dans le champ du discours rapporté. L'Information grammaticale, 55, 38-42.

Authier-Revuz, J. (1993). Repères dans le champ du discours rapporté (suite). L'Information grammaticale, 56, 10-15.

Banfield, A. (1973). Narrative style and the grammar of direct and indirect speech. Foundations of Language, 10, 139.

Banfield, A. (1979). Où l'épistémologie, le style et la grammaire rencontrent la théorie littéraire. Langue française, $44,9-26$.

Banfield, A. (1982). Unspeakable Sentences: narration and representation in the language of fiction. Boston: Routledte \& Keagan Paul.

Charaudeau, P. et Maingueneau, D. (2002). Dictionnaire d'analyse du discours. Paris : Seuil.

Cohn, D. C. (1984). Transparent Minds. Narrative Modes for Presenting Consciousness in Fiction. Princeton: Princeton University Press.

Dubois, B. L. (1989). Pseudoquotation in Current English Communication; "Hey, she didn't really say it.". Language in society, 18, 343-359.

Grevisse, M. et Goosse, A. (1993). Le bon usage : grammaire française. Paris : Duculot.

Hosaka, M. (1981). Die Erlebte Rede im Japanischen. Bulletin of College of General Education of Ibaraki University. 13, 95-109.

Imprimerie nationale. (2002). Lexique des règles typographiques en usage à l'Imprimerie nationale. Paris: Imprimerie nationale.

Kamada, O. (2000). Nihongo no In'yô. Tokyo : Hituzi Syobo.

Li, C. N. et Thompson, S. A. (1976). Subject and Topic: a New Typology of Language. In Li, C. N. (ed.) Subject and Topic. New York: Academic Press, 457-489.

Maingueneau, D. (2000). Instances frontières et angélisme narratif. Langue française, 128, 74-95.

Maingueneau, D. (2005). Linguistique pour le texte littéraire. Paris : Armand Colin.

Maingueneau, D. (2010). Manuel de linguistique pour les textes littéraires. Paris : Armand Colin.

Nakayama, M. (1990). Sur le « point de vue » dans le texte narratif. Humanities review, 16, 41-54.

Nakayama, M. (1993). Quand écrire, c'est faire : les romans d'Abe Kôbô et leur traduction. Essays and Studies vol. 44 no. 1, 1-31.

Perrousseaux Y. (2005). Manuel de typographie française élémentaire. Méolans-Revel : Atelier Perrousseaux Éditeur. 
Philippe, G. (1997). Le discours en soi. La représentation du discours intérieur dans les romans de Sartre. Paris : Éditions Champion.

Philippe, G. (2001). Le paradoxe énonciatif endophasique et ses premières solutions fictionnelles. Langue francaise, $132,96-105$

Rabatel, A. (1998). La construction textuelle du point de vue. Lausanne et Paris : Delachaux et Niestlé.

Rabatel, A. (2001). Les représentations de la parole intérieure monologue intérieur, discours direct et indirect libres, point de vue. Langue francaise, 132, 72-95.

Rabatel, A. (2004). Les verbes de perception, entre point de vue représenté et discours représentés. In Manuel, J., Muñoz, L., Marnette, S. et Rosier, L. (éd.) Le discours rapporté dans tous ses états : actes du colloque international, Bruxelles, 8-11 novembre 2001. Paris : L'Harmattan, 81-93.

Rabatel, A. (2004). L'effacement énonciatif dans les discours rapportés et ses effets pragmatiques. Langages, 156, 117 .

Rabatel, A. (2005). Effacement énonciatif et argumentation indirecte : «On-perceptions », " on-représentations » et " on-vérités » dans les points de vue stéréotypés. In Raccah, P.-Y. (éd.) Signes, langues et cognition. Paris : L'Harmattan, 85-116.

Risterucchi-Roudnicky, D. (2008). Introduction à l'analyse des æuvres traduites. Paris : Armand Colin.

Rosier, L. (1999). Le discours rapporté : histoire, théories, pratiques. Paris : Duculot.

Rosier, L. (2008). Le discours rapporté en français. Paris : Ophrys.

Takagaki, Y. (2010). Les noms de titre familial comme marqueurs de cohérence. In Neveu, F., Muni Toke, V., Klingler, T., Durand, J., Mondada, L., et Prévost S. (éd.) $2^{e}$ Congrès Mondial de Linguistique Française, 12151225 ,

$<$ http://www.linguistiquefrancaise.org/index.php?option=com_toc\&url=/articles/cmlf/abs/2010/01/contents/conte nts.html>.

Takagaki, Y. (2011). De la rhétorique contrastive à la linguistique textuelle : l'organisation textuelle du français et $d u$ japonais. Rouen et Osaka: Publications des Universités de Rouen et du Havre et Osaka Municipal Universities Press.

Wilmet, M. (2003). Grammaire critique du français. Bruxelles : Duculot.

Zribi-Hertz, A. (1989). Anaphor binding and narrative point of view: Englilsh reflexive pronous in sentence and discourse. Language, 65-4, 695-727.

Zribi-Hertz, A. (1990). Lui-même argument et le concept de «pronom A ». Langages, 97, 100-127.

\footnotetext{
${ }^{1}$ Traduction d'Alain Rocher. Natsume Sôseki, Dix rêves, In Anthologie de nouvelles japonaises contemporaines, Tome II, Paris, Gallimard, 1989.

${ }^{2}$ Dans la terminologie de Maingueneau, $M Q C$ signifie « membre quelconque d'une collectivité ».

${ }^{3} C f$. note 2 .

${ }^{4} \mathrm{Si}$ le verbe penser est dans cette liste, c'est pour ne pas exclure les paroles intérieures dans notre analyse. $C f$. exemples (5) et (6a).

${ }^{5}$ Dans notre corpus de textes littéraires, le discours direct avec guillemets sans verbe introducteur est plus fréquent en japonais qu'en français. En japonais, il existe aussi une autre forme plus insolite : une phrase dont la première moitié est en discours direct mis entre guillemets et le reste en discours indirect sans guillemets.

${ }^{6}$ Traduction d'Anne et Cécile Sakaï. Yûko Tsushima, Territoire de la lumière, Paris, Des femmes, 1986.

${ }^{7}$ L'emploi de cette expression fournit également d'autres informations : il s'agit d'une conversation décontractée avec un égal ou un inférieur.

${ }^{8}$ Traduction de Dominique Palmé et Kyôko Satô. Banana Yoshimoto, Kitchen, Paris, Gallimard, 1994.

${ }^{9}$ Traduction de René Sieffert. Yasunari Kawabata, Les Belles endormies, Paris, Albin Michel, 1982.
} 
${ }^{10}$ Le texte original japonais ne contient pas de verbe introducteur, mais l'expression correspondante à " Qu'est-ce que c'est? " est suivie d'une marque de citation to, qui correspondrait en français à que. La présence de ce mot suggère qu'il y a un acte d'énonciation. Sans ce mot to, le texte japonais serait bizarre.

${ }^{11}$ Exemple cité par Nakayama (1990). Traduction de Pierre Faure. Kafû Nagai, La Sumida, Paris, Gallimard, 1975.

12 Traduction de Georges Bonneau. Abé Kôbô, La Femme des sables, Paris, Stock, 1979 (1990).

${ }^{13}$ Cf. note 12 . 